\title{
MicroRNAs, a subpopulation of regulators, are involved in breast cancer progression through regulating breast cancer stem cells (Review)
}

\author{
XUEMEI FAN ${ }^{1 *}$, WEI CHEN ${ }^{2 *}$, ZIYI FU $^{1}$, LIHUA ZENG $^{1}$, YONGMEI YIN ${ }^{3}$ and HONGYAN YUAN ${ }^{1,4}$ \\ ${ }^{1}$ Nanjing Maternity and Child Health Medical Institute, Obstetrics and Gynecology Hospital \\ Affiliated to Nanjing Medical University, Nanjing, Jiangsu 210004; ${ }^{2}$ Department of Head and Neck Surgery, \\ Jiangsu Cancer Hospital Affiliated to Nanjing Medical University, Nanjing, Jiangsu 210000; ${ }^{3}$ Department of Oncology, \\ First Affiliated Hospital, Nanjing Medical University, Nanjing, Jiangsu 210029, P.R. China; ${ }^{4}$ Department of Oncology, \\ Lombardi Comprehensive Cancer Center, Georgetown University Medical Center, Washington, DC 20007, USA
}

Received December 20, 2015; Accepted May 18, 2017

DOI: $10.3892 / 01.2017 .6867$

\begin{abstract}
Cancer stem cells (CSCs; also known as tumor-initiating cells) are essential effectors of tumor progression due to their self-renewal capacity, differentiation potential, tumorigenic ability and resistance to chemotherapy, all of which contribute to cancer relapse, metastasis and a poor prognosis. Breast cancer stem cells (BCSCs) have been identified to be involved in the processes of $\mathrm{BC}$ initiation, growth and recurrence. MicroRNAs (miRNAs) are a class of non-coding small RNAs of 19-23 nucleotides in length that regulate gene expression at the post-transcriptional level through various mechanisms, and serve critical roles in cancer progression. miRNAs have been demonstrated to elicit effects on BCSCs characteristics via the targeting of oncogenes or tumor suppressor genes. The present study focused on the effect of miRNAs on BCSC, including BCSC formation, self-renewal and differentiation, by which miRNAs may inhibit BCSC invasion and metastasis, modulate clonogenicity and tumorigenicity of BCSCs as well as regulate chemotherapy resistance to $\mathrm{BC}$. Through an improved understanding of the association
\end{abstract}

Correspondence to: Dr Hongyan Yuan, Nanjing Maternity and Child Health Medical Institute, Obstetrics and Gynecology Hospital Affiliated to Nanjing Medical University, 123 Tianfei Street, Mochou Road, Nanjing, Jiangsu 210004, P.R. China

E-mail:dryhy@hotmail.com

Dr Yongmei Yin, Department of Oncology, First Affiliated Hospital, Nanjing Medical University, 300 Guangzhou Road, Nanjing, Jiangsu 210029, P.R. China

E-mail: yinyongmei_sr@sina.com

${ }^{*}$ Contributed equally

Key words: cancer stem cells, microRNAs, breast cancer, stemness, metastasis, tumorigenicity, chemotherapy resistance between BCSCs and miRNAs, a novel and safer therapeutic target for BC may be identified.

\section{Contents}

1. Introduction

2. miRNAs participate in BCSC formation

3. miRNAs regulate BCSC self-renewal

4. miRNAs mediate BCSC differentiation

5. miRNAs inhibit BCSC invasion and metastasis

6. miRNAs modulate clonogenicity and tumorigenicity of BCSCs

7. miRNAs regulate chemotherapy resistance to BC by modulating BCSC traits

8. Prospects

9. Conclusion

\section{Introduction}

Previous studies have provided support for the hypothesis that breast cancer (BC) development is driven by a subpopulation of cells that exhibit stem cell characteristics, such as a capability for self-renewal, differentiation, metastasis, tumorigenicity and intrinsic resistance to chemotherapy (1). This subpopulation of cells is recognized as breast cancer stem cells (BCSCs), which are essential for BC progression $(2,3)$. MicroRNAs (miRNAs/miRs) are small non-coding RNAs that regulate multiple signaling pathways and affect cancer progression through targeting associated genes. miRNAs may induce degradation or restrain translation of their target mRNAs by binding to the 3 ' untranslated region (UTR) $(4,5)$. miRNAs have been implicated in tumor progression and therapeutic resistance; however, the molecular mechanisms that define this state remain unclear (6). Dysregulation of miRNAs participating in $\mathrm{BC}$ progression, inlcuding oncogenesis, apoptosis, proliferation, metastasis, invasion and even drug resistance (7). Increasing evidence suggests that miRNAs may participate 
in BC progression through altering the stemness of BCSCs, which primarily involves tumor formation, self-renewal, differentiation, metastasis, tumorigenicity and chemotherapy resistance $(8,9)$. Therefore, BCSCs may be potential targets for miR-based therapy.

\section{2. miRNAs participate in BCSC formation}

BCSCs may be identified and isolated according to their cell surface markers, including the phenotype of $\mathrm{CD} 44^{+} \mathrm{CD} 24^{-}$and ALDH1+ $(10,11)$. miRNAs are involved in tumor biology by regulating associated genes, and their roles in BCSC formation are becoming known: Tumor suppressor tumor protein p53 (p53) transactivates miR-200c and serves a role in reducing the CD $44^{+} \mathrm{CD} 24^{-}$stem cell population through directly binding to the miR-200c promoter and increasing expression of miR-200c (12). Endoribonuclease dicer (DICER), an enzyme involved in microRNA processing, is suppressed by hypoxia through silencing of the expression of the DICER promoter (13). Subsequently, decreased miRNA processing leads to expression of the miR-200 target zinc finger E-box binding homeobox 1 (ZEB1), which in turn causes an epithelial-mesenchymal transition (EMT)-driven acquisition of stem cell properties in BC (13). Sine oculis homeobox homolog 1 (Six1), a metastatic regulator, was suggested to activate the tumor-promotional arm of transforming growth factor- $\beta$ (TGF- $\beta$ ) signaling via increasing the expression of the miR-106b-25 cluster. Upregulated miR-106b- 25 by Six 1 promotes TGF- $\beta$-mediated upregulation of $\mathrm{CD} 44^{+} \mathrm{CD} 24^{-}$BCSCs through targeting the inhibitory mothers against decapentaplegic homolog (Smad) 7 protein of TGF- $\beta$ signaling (14). The miR-140/aldehyde dehydrogenase 1 family member $1 \mathrm{~A}(\mathrm{ALDH} 1) / \mathrm{sex}$ determining region Y-Box (SOX)9 axis also serves an important role in BCSC formation in vivo. miR-140 is downregulated in ductal carcinoma in situ (DCIS) stem-like cells, and inhibits CSC formation in basal-like early-stage BC. miR-140 reduces BCSC formation by targeting SOX9 and ALDH1, which have the highest level of activated CSC factors in DCIS stem-like cells (15). mir-34a suppresses BCSC characteristics at least partly through inhibiting Notch1 expression. Notch1 expression decreased by mir-34a was identified to decrease the percentage of $\mathrm{CD} 44^{+} \mathrm{CD} 24^{-}$cells and the expression of ALDH1 (16). Ligand jagged1 is secreted from the tumor stroma to promote the BCSC phenotype through repressing the expression of miR-205. Hairy and enhancer of split-1, as a transcription repressor activated by Jagged1-Notch1 signaling, is involved in the inhibition of miR-205 expression (17). Decreased miR-205 increases the BCSC population ratio through significantly promoting the proportion of the CSCs population that exhibits the $\mathrm{CD} 44^{+} \mathrm{CD} 24^{-}$phenotype (17). In addition, Notch2, as a target of miR-205 and also activated by loss of miR-205, is involved in CSC stemness through increasing the $\mathrm{CD} 44^{+} \mathrm{CD} 24^{-}$cell population (15). Ectopic expression of miR-7 significantly decreases the percentage of CD 44 ${ }^{+} \mathrm{CD} 24^{-}$cells in MDA-MB-231 cells. miR-7 decreases the BCSC population in BC partly by the downregulation of the signal transducer and activator of transcription 3 (STAT3) pathway via inhibiting the expression of SET domain bifurcated 1 (SETDB1) (18). Krüppel-like factor (KLF) 8-induced expression of miR-146a was suggested to account for the acquisition of BCSC traits, due to its effect on increasing the $\mathrm{CD}_{4} 4^{+} \mathrm{CD} 24^{-}$and $\mathrm{ALDH}^{+}$expression levels. miR-146a mediates KLF8-induced CSC features by inhibiting the expression of the Numb homolog (NUMB), a Notch signaling inhibitor (19). miR-21 was identified to increase the proportion of BCSCs that expressed the CSC surface biomarkers CD $44^{+} \mathrm{CD} 242^{-}$and $\mathrm{ALDH}^{+}(20)$. miR-21 induces the BCSC phenotype through the depletion of phosphatase and tensin homolog and the activation of protein kinase B (AKT) and extracellular signal-related kinase 1/2 (20). miRNA-125a-targeted leukemia inhibitory factor receptor changes the activity of transcriptional co-activator with PDZ-binging motif, an effector molecule in the Hippo pathway, through which miRNA-125a increases the percentage of stem cells in MCF7 cells (21). Increased miR-34c inhibits the development of $\mathrm{CD} 44^{+} \mathrm{CD} 24^{-}$and $\mathrm{ALDH}^{+}$cells in the $\mathrm{BC}$ cell population by targeting Notch4 (22). Progesterone (P4) contributes to the expansion of stem-like breast cancer cells through decreasing the level of miR-141, a member of the miR-200 family of tumor suppressors, which directly targets STAT5A and progesterone receptor (PR) (Fig. 1) (23).

\section{3. miRNAs regulate BCSC self-renewal}

As a characteristic feature of stem cells, self-renewal ensures that BCSCs survive for long periods of time. miRNAs, including miR-145, miR-128b, miR-15/16 (miR-16, miR-15b), and the miR-103/107 (miR-103, miR-107) and miR-200 (miR-200b, miR-200a, miR-429, miR-200c) families, were identified to be involved in the mammosphere formation of BCSCs. Individual upregulation of these miRNAs restrains the formation of mammospheres by at least $50 \%$. The miR-200 family directly targets the stem cell transcription factor KLF4, enhancer of zeste 2 polycomb repressive complex 2 subunit (EZH2) and polycomb complex protein BMI1 (BMI1) (24). Additionally, miR-200 also targets and inhibits the suppressor of zeste 12 (SUZ12) (25) and BMI1 (26), which, respectively, are are subunits of the polycomb repressive complex (PRC) 2 and PRC1 that repress transcription. miR-200 target genes may also be regulated by other miRNAs that are reduced in BCSCs and essential for BCSC formation (27). For example, ZEB2 and KLF4 are putative targets of miR-145, BMI1 is a putative target of miR-128b, and SUZ12 is a putative target of the miR-103/107 and miR-15b/16 families (24). Thus, we can conclude that the expression of the CSC-modulating miRNAs, including miR-200b, miR-15b, miR-128b, miR-107 and miR-145, is inhibited by ZEB1 and ZEB2. In addition, TGF- $\beta$ expression synergizes with RAC- $\alpha$ serine/threonine-protein kinase-knockdown in promoting mammosphere formation through a decrease in the abundance of miR-200 (28). miR-16 inhibits the mammosphere-forming ability of mammary tumor cells by regulating wild-type p53-induced phosphatase 1 (WIP1) induction in the DNA damage response through targeting the 3'UTR of WIP1 (29). Pleckstrin homology-like domain, family A, member1 (PHLDA1) in mammospheres, inhibited by miR-181a/b, leads to attenuated mammosphere formation in estrogen receptor $(\mathrm{ER})^{+} \mathrm{BC}$. Additionally, crosstalk between ER and the nuclear factor $\kappa$-light-chain-enhancer of activated B cells pathway contributes to the upregulation of PHLDA1, directly 


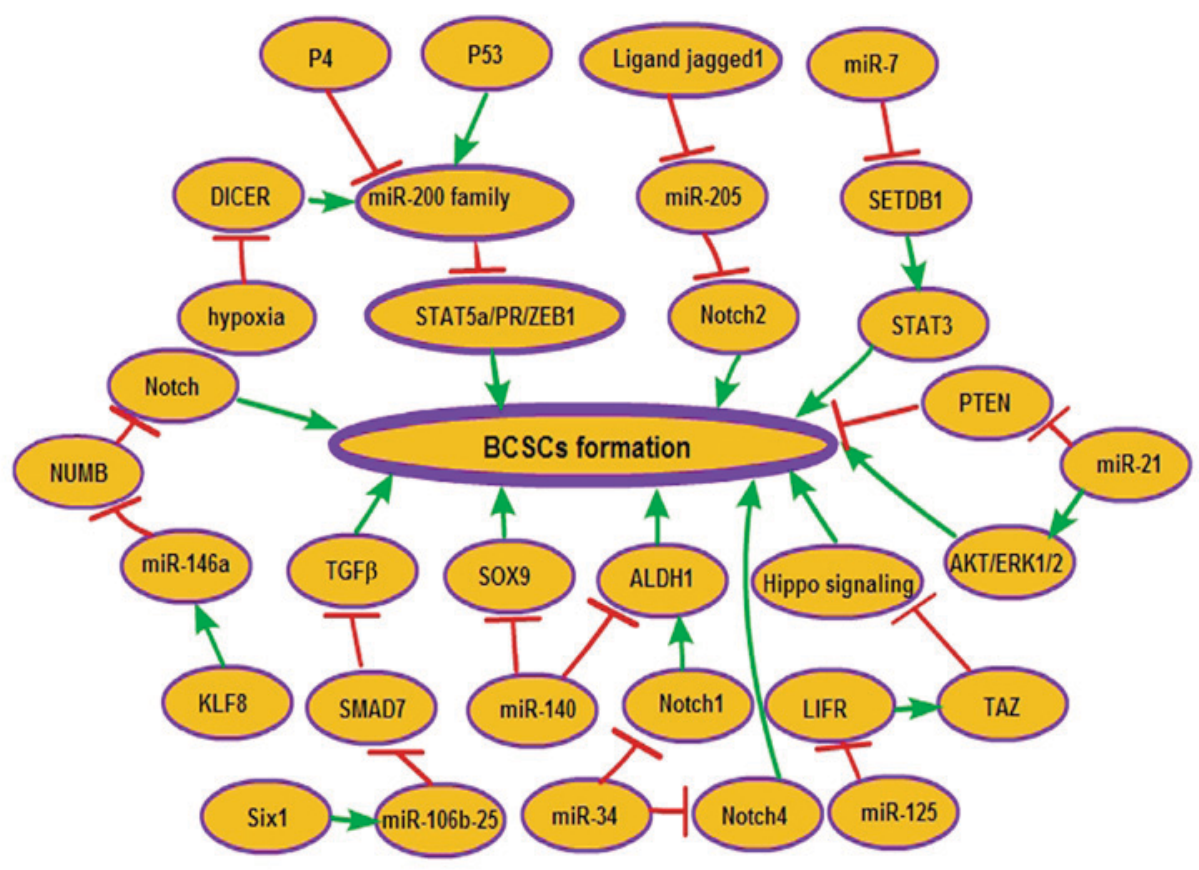

Figure 1. miRNAs participate in BCSC formation. The formation of BCSCs is regulated by multiple signaling pathways, including Notch1/2, STAT3/5, Hippo, ERK1/2 and TGF- $\beta$ signaling. miRNAs, including miR-200, miR-205, miR-7, miR-21, miR-125, miR-140, miR-106b-25 and miR-146a, modulate BCSC formation through activation (green line) or inactivation (red line) of these signaling pathways. Additionally, the expression of miRNAs is associated with various genes that include P4, P53, DICER, KLF8, Six1 and Ligand jagged1. miR/miRNA, microRNA; BCSC, breast cancer stem cell; STAT3/5, signal transducer and activator of transcription 3/5; ERK, extracellular signal-related kinase; TGF- $\beta$, transforming growth factor- $\beta$; P4, progesterone; $p 53$, tumor protein 53; DICER, endoribonuclease dicer; NUMB, numb homolog; KLF8, Krüppel like factor 8; SMAD7, mothers against decapentaplegic homolog 7; Six1, sine oculis homeobox homolog 1; SOX9, SRY-Box 9; ALDH1, aldehyde dehydrogenase 1 family member 1A; PTEN, phosphatase and tensin homolog; Akt, protein kinase B; TAZ, transcriptional co-activator with PDZ-binging motif; LIFR, leukemia inhibitory factor receptor; SETDB1, SET domain bifurcated 1; $\mathrm{PR}$, progesterone receptor.

through the increased transcription and indirectly through the inhibition of miR-181a/b (30). Estrogen (E2) was identified to enhance breast tumor-initiating cell survival by downregulating miR-140, which targets SOX2 (31). Concomitantly, the transcription of miR-140 was also inhibited by estrogen receptor $\alpha(E R \alpha)$, which binds to the promoter of miR-140; reduced miR-140 increases breast tumor-initiating cell renewal via targeting SOX2 (32). In addition, miR-140 serves a critical role in regulating stem cell signaling in basal-like DCIS. miR-140 overexpression reduces stem cell renewal and tumor growth in vivo through directly targeting ALDH1 and SOX9, the stem-cell factors with the highest expression level in basal-like DCIS stem cells (15). Upregulated mir-93 inhibits several stem cell regulatory genes, including STAT3, Janus kinase 1, high mobility group AT-hook 2 (HMGA2), enhancer of zeste 1 polycomb repressive complex 2 subunit, SOX4 and RAC- $\gamma$ serine/threonine-protein kinase, through which miR-93 results in the depletion of BCSCs (33). Side population (SP) cells exhibit characteristics similar to CSCs (34). It was suggested that miR-99a reduces the self-renewal capacities of BC SP cells in vivo through activating mammalian target of rapamycin (mTOR), a downstream effector of the AKT/phosphoinositide 3-kinase (PI3K) signaling pathway (35). Ectopic expression of miR-34c inhibits the self-renewal of BCSCs and suppresses tumor growth by targeting and silencing expression of Notch4 (21). Cyclo-oxygenase (COX)-2 promotes the BCSC phenotype by increasing the expression of miR-526b, owing to the activation of the prostaglandin E2 receptor EP4 and downstream PI3K/AKT and protein kinase A signaling pathways (36). Ectopic miR-526b increases the number and size of spheroids, which suggests that upregulated miR-526b is associated with the stimulation of BCSCs (36).

\section{4. miRNAs mediate BCSC differentiation}

The balance between self-renewal and differentiation is an additionally important characteristic of BCSCs, and multiple miRNAs have been suggested to participate in regulating this balance. In the $\mathrm{CD} 44^{+}$cell population, miR-29 members are downregulated by $\mathrm{P} 4$, which promotes the expansion of stem-like cancer cells in $\mathrm{ER}^{+}$and $\mathrm{PR}^{+} \mathrm{BC}$. Concurrently, downregulated miR-29 members also enhance the expansion of $\mathrm{CD}_{4} 4^{+}$and $\mathrm{CK}^{+}$cells in response to $\mathrm{P} 4$ (37). The reprogramming of differentiated cells into pluripotent stem cells and the maintenance of BCSCs are inhibited by miR-29 members that target KLF4 (37). Induced expression of miR-200c promotes differentiation of claudin-low tumors in vivo by increasing the expression of basal and luminal markers, specifically keratins K14 and K8 (27). Notably, the differentiation is more similar to the differentiated basal-like tumors compared with the undifferentiated claudin-low tumors from which they originated (27). miR-200c alters the functionality of BCSCs through exhibiting the expression of stem cell-associated genes BMI1 and EZH2, and increases the levels of differentiation markers GATA binding protein and E74-like factor 5, which results in a more differentiated 


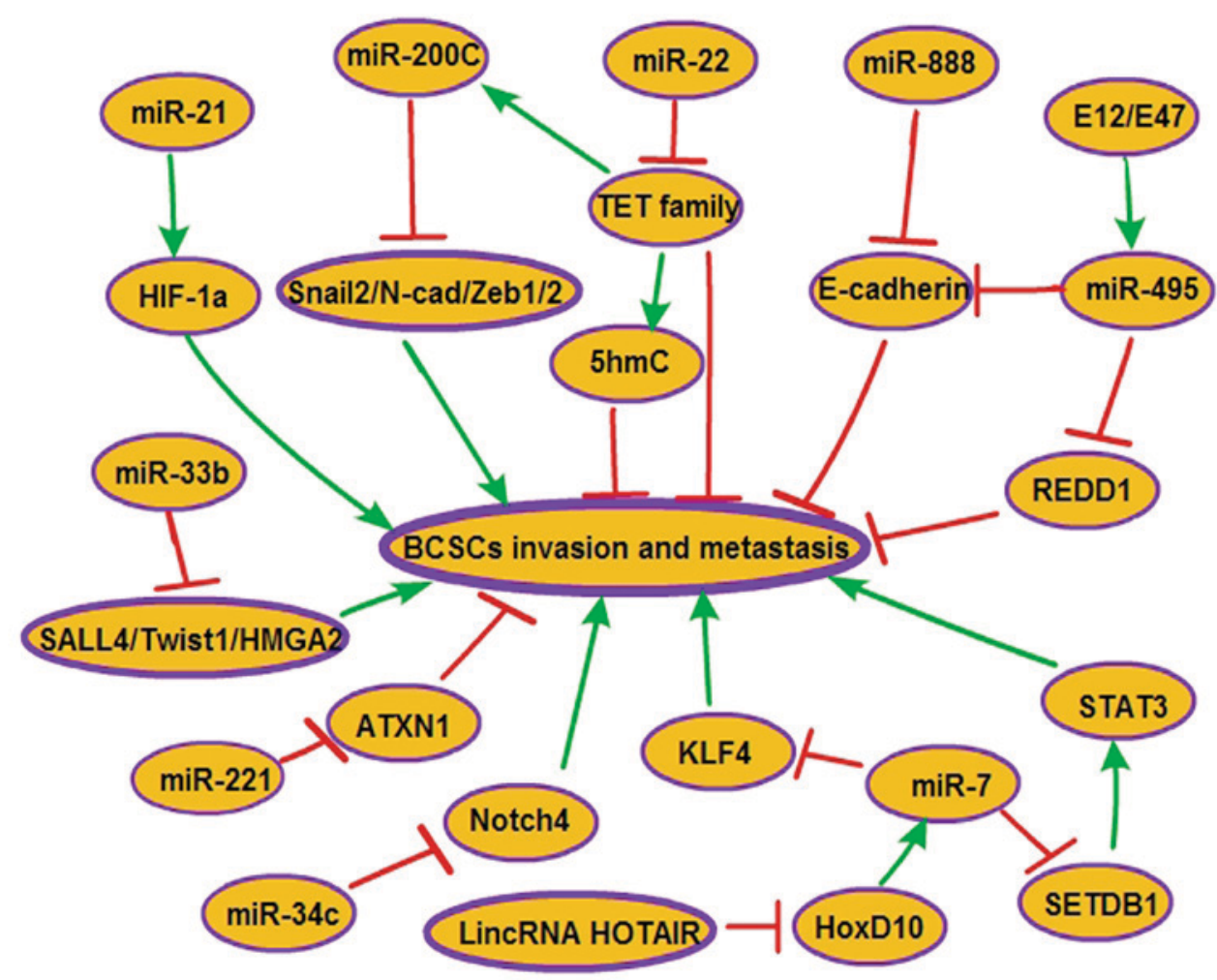

Figure 2. miRNAs inhibit BCSC invasion and metastasis. miRNAs, including miR-21, miR-22, miR-33b, miR-34c, miR-7, miR-221, miR-200c, miR-495 and miR-888, serve important roles in regulating the invasion and metastasis of BCSCs through modulating the expression of oncogenes or anti-oncogenes. Decreased levels of expression of anti-oncogenes, which include E-cadherin, REDD1, ATXN1, the TET family and 5hmC, or increased levels of expression of oncogenes, such as HIF-1 $\alpha$, SALL2/4, Twist1, HMGA2, ZEB1/2, NOTCH4, KLF4 and STAT3, by miRNAs were suggested to promote BCSC invasion and metastasis. Additionally, miR-7 and miR-495 as onco-miRNAs may be regulated by HoxD10 and E12/E47, respectively. miR/miRNA, microRNA; BCSCs, breast cancer stem cells; E-cadherin, epithelial cadherin; N-cad, N-cadherin; REDD1, DNA damage-inducible transcript 4 protein; ATXN1, ataxin-1; 5hmC, 5-hydroxymethylcytosine; TET, ten-eleven translocation; HIF-1 $\alpha$, hypoxia-inducible factor 1 $\alpha$; SALL2/4, sal-like protein 2/4; Twist1, twist-related protein 1; HMGA2, high mobility group AT-hook 2; ZEB1/2, zinc finger E-box binding homeobox 1/2; NOTCH4; KLF4, Krüppel-like factor 4; STAT3, signal transducer and activator of transcription 3; lincRNA, long intergenic non-coding RNA; HOTAIR, homeobox transcript antisense RNA; HoxD10, homeobox D10; SETDB1, SET domain bifurcated 1; SNAIL2, zinc finger protein SNAI2.

status of claudin-low tumors in vivo (27). miR-200c may also induce differentiation of BCSCs by targeting BMI1 (38). miR-100 serves a pivotal role in modulating differentiation of patient-derived basal-like BCSCs (39). Upregulated miR-100 interferes with the properties of BCSCs, and alters the basal-like phenotype into a more differentiated luminal phenotype, via inhibiting polo-like kinase 1 (Plk1), SWItch/sucrose non-fermentable-related, matrix-associated, actin-dependent regulator of chromatin, and the Wnt/ $\beta$-catenin signaling pathway (39).

\section{5. miRNAs inhibit BCSC invasion and metastasis}

Invasion and metastasis remain the most complex and challenging problems of $\mathrm{BC}$ treatment and prognosis. EMT, which is assessed by the decreased expression of epithelial cell markers [keratins and epithelial (E)-cadherin] and the increased expression of mesenchymal cell markers $[\alpha$-smooth muscle actin ( $\alpha$-SMA), vimentin and $\mathrm{N}$-cadherin], contributes to invasion and metastasis in BC and is significantly associated with the acquisition of BCSC characteristics (40). Previous evidence has demonstrated that multiple miRNAs are also involved in the metastasis process of $\mathrm{BC}$ through inhibiting BCSC functionality. Han et al revealed that miR-21 and hypoxia-inducible factor- $1 \alpha(\mathrm{HIF}-1 \alpha)$ are upregulated in the third-sphere forming (3-S) CSC-like cells, which are isolated from MCF-7 parental cells and exhibit high levels of CSC surface markers (CD44 ${ }^{+} / \mathrm{CD} 24^{-/ \text {low }}$ and $\left.\mathrm{ALDH} 1^{+}\right)$. Antagonism of miR-21 reverses EMT and impedes invasion and migration in the 3-S CSC-like cells via HIF-1 $\alpha$ downregulation (41). In addition, miR-21 re-expression promotes the process of migration and invasion by enhancing the characteristics of CSCs and activating the EMT process in BC MCF-7 cells (42). As an important regulator of EMT, the upregulation of the miR-200 family reverses EMT and reduces metastatic potential in claudin-low breast cancer, which is significantly enriched in BCSCs, via the downregulation of ZEB1/2, zinc finger protein SNAI2, N-cadherin and transcriptional repressors of E-cadherin (27). It was demonstrated that miR-22 expands BCSC in size, and enhances cell invasion and metastasis in a BC mouse xenograft through its ability to repress the expression of miR-200 and 5-hydroxymethylcytosine $(5 \mathrm{hmC})$ by directly targeting members of the ten-eleven translocation (TET) family (43). miR-33b acts as a negative regulator of $\mathrm{BC}$ stem-like cell self-renewal, migration and invasion in highly metastatic BC cells, and represses lung metastasis in vivo by targeting its downstream targets, including sal-like protein 4 , twist-related protein 1 and 
Table I. miRNAs regulate chemotherapy resistance in BC by modulating BC stem cell traits.

\begin{tabular}{lll}
\hline miRNA & \multicolumn{1}{c}{ Target gene } & \multicolumn{1}{c}{ Function } \\
\hline miR-200c & BMI1 & Increase 5-fluorouracil sensitivity \\
miR-100 & Polo-like kinase 1 & Increase hormonal sensitivity \\
miR-146a & Numb homolog 1 & Promote paclitaxel resistance \\
miR-34a & HDAC1, HDAC7 & Increase paclitaxel/doxorubicin/cisplatin sensitivity \\
miR-34a & Notch1 & Increase paclitaxel sensitivity \\
miR-27b & Ectonucleotide pyrophosphatase/ & Increase docetaxel sensitivity \\
miR-125b/205/424 & Phosphodiesterase 1 & \\
& Protein kinase B/mechanistic & Promote aromatase inhibitor resistance \\
miR-128 & target of rapamycin & \\
BMI1/ATP-binding cassette & Increase doxorubicin sensitivity \\
miR-16 & subfamily C member 5 & \\
& Wild-type p53-induced & Increase doxorubicin sensitivity \\
\hline
\end{tabular}

miR/miRNA, microRNA; BC, breast cancer; BMI1, polycomb complex protein BMI1; HDAC, histone deacetylase; ATP, adenosine 5'-triphosphate.

HMGA2 (44). miR-888 was identified to act as a repressor of the adherens junction pathway and serve a critical role in maintaining SP properties and regulating EMT, invasion and metastasis in MCF-7 SP cells via directly targeting E-cadherin (45). Increases in levels of miR-495 enriched in $\mathrm{PROCR}^{+} / \mathrm{ESA}^{+}$and $\mathrm{CD} 44^{+} / \mathrm{CD} 24^{-/ \text {low }} \mathrm{BCSC}$ subpopulations are upregulated by E12/E47 (46). miR-495 overexpression maintains BCSC properties such as promotion of metastasis and invasion via suppressing E-cadherin and DNA damage-inducible transcript 4 protein (REDD1) (46). The overexpression of miR-221 is able to stimulate stem-like properties in the luminal type of BC cells and induce EMT in BC cells through downregulating ataxin-1 (47). miR-34c reduction via DNA methylation in breast tumor-initiating cells (BT-Ics) promotes self-renewal, EMT and migration of BT-ICs by targeting Notch4 (22). miR-7 suppresses brain metastasis of BCSCs in vivo by downregulating the critical downstream target KLF4, an induced pluripotent stem cell gene that is important for the maintenance of stemness of progenitor cells (48). Additionally, miR-7 was also demonstrated to reduce the size of the BCSC population, partially reverse EMT in MDA-MB-231 cells and repress the metastasis of BCSCs in adrenal glands, kidneys and lungs in non-obese diabetic/severe combined immune deficiency (NOD/SCID) mice by directly targeting the 3'UTR of SETDB1, which serves a key role in activating the STAT3 pathway. In addition, long intergenic non-coding RNA homeobox (HOX) transcript antisense RNA indirectly inhibits miR-7 via downregulating the expression of homeobox D10 (18) (Fig. 2).

\section{6. miRNAs modulate clonogenicity and tumorigenicity of BCSCs}

miRNAs are considered to be potential biomarkers or therapeutic targets of $\mathrm{BC}$, due to their capability of modulating stem cell biology, including clonogenicity and tumorigenicity. miR-526b, a COX-2-upregulated oncogene, promoted tumorsphere formation in BC cells and lung colony formation in an experimental metastasis model, relying on EP4 receptor activity and cyclic adenosine monophosphate (CAMP) and downstream PI3K/AKT signaling pathways (36). In addition, miR-495 that is upregulated by transcription factor E2A immunoglobulin enhancer-binding factors E12/E47, directly represses E-cadherin and REDD1, and contribute to an increase in BCSC traits and hypoxia resistance, which then promotes colony formation in $\mathrm{BC}$ cells and tumorigenesis in mice (46). Progestins significantly increase mammosphere formation in vitro and enhance the tumor-initiating capability in hormone-responsive breast cancer via repressing miR-29, to augment the PR-mediated upregulation of KLF4 (15). The glabridin (GLA)/miR-148a/SMAD2 axis serves a critical role in modulating CSC-like properties, such as the formation of mammospheres and colonies. GLA-upregulated miR-148a results in a repression of clone formation, as miR-148a is able to inhibit endogenous TGF- $\beta$ /SMAD2 signaling in BC cells (49). It was identified that miR-99a directly inhibits the mTOR signaling pathway in breast cancer SP cells, which results in the suppression of tumorigenicity in vivo (35). In addition, miR-200c that targets BMI1, suppresses clonogenicity and tumorigenicity of BCSCs in NOD/SCID mice due to the inhibition of self-renewal and proliferation of BCSCs (38). Conversely, miR-22, an oncogene, is able to promote tumorigenesis in transgenic mice through expanding the BCSCs in size (43). The overexpression of miR-22 represses the expression of miR-200 s and $5 \mathrm{hmC}$ by targeting members of the TET family (43). miR-128-2, embedded in the intron of the CAMP-regulated phosphoprotein 21 gene at chromosome 3 p22.3, serves critical roles in the modulation of oncogenic transformation and progression in mammary epithelial cells (50). miR-128-2 is downregulated by TGF- $\beta$ through the phosphorylation of TGF- $\beta 1$ receptor to enhance a specific SNAIL protein expression. In addition, miR-128-2, downregulated by SNAIL, promotes mammary epithelial oncogenic transformation via expressing a group of direct targets 
(colony-stimulating factor 1, BMI1, Lin-28 homology A, nanog homeobox and KLF4), which together act to activate the STAT3 and PI3K/AKT signaling pathways (50).

\section{7. miRNAs regulate chemotherapy resistance to $\mathrm{BC}$ by modulating BCSC traits}

Chemotherapy resistance in $\mathrm{BC}$ is one of the major obstacles for clinical intervention, and one of the hallmarks of BCSCs. An increasing number of studies have suggested the key role of miRNAs in chemoresistance by regulating BCSC traits (51). Cross-talk between miR-200c and BMI1, modulated by p53, and BMI1 repression in breast cancer cells promotes the sensitivity of $\mathrm{BC}$ to 5-fluorouracil through reducing the proportion of $\mathrm{CD}_{4} 4^{+} / \mathrm{CD} 24^{-}$cells in the BCSC population, and inducing susceptive apoptosis (52). The differentiation process, triggered by miR-100, which attenuates BCSC properties and promotes the basal like phenotype into a more differentiated luminal phenotype in patient-derived basal-like BCSCs, induces the expression of ER and sensitizes basal-like BCSCs to hormonal therapy via downregulating PLK1 (39). KLF8 serves a critical role in regulating the induction and maintenance of BCSC traits, which contributes to the resistance of cells to the cytotoxic effect of paclitaxel in MCF-10A cells via targeting miR-146a that binds to the 3'-UTR of NUMB and inhibits NUMB expression (19). It was confirmed that histone deacetylase (HDAC)1 and HDAC7 are downstream targets of miR-34a and are upregulated in the $\mathrm{CD} 44^{+} \mathrm{CD} 24^{-}$subpopulation (53). Deacetylation of acetyl-heat shock protein 70 (HSP70; K246) by HDAC7 and HDAC1 increases resistance to therapeutics [paclitaxel (PTX), doxorubicin and cisplatin] through the inhibition of autophagy in MCF-7 cells expressing wild-type HSP70 (53). Furthermore, the overexpression of miR-34a that targets Notch1 also enhances chemosensitivity to PTX by suppressing the proliferation of BCSCs (16). Metformin, the anti-type II diabetes (T2D) drug, was identified to decrease the generation of SPs in BC cells, leading to an attenuation in chemoresistance to docetaxel and tumor-seeding ability through miR-27b-mediated inhibition of ectonucleotide pyrophosphatase/phosphodiesterase 1 (ENPP1). Uninhibited ENPP1 enhances the generation of SPs via upregulating the adenosine 5'-triphosphate (ATP) cassette sub-family $\mathrm{G}$ member 2 transporter (54). miR-125b, as a positive regulator of SP and CSC properties in BC cell lines and primary BC cells, contributes to chemoresistance to paclitaxel (55). Additionally, ectopic overexpression of miR-205 or miR-125b and silencing miR-424 expression are sufficient to induce a subpopulation of cells that exhibit stem-like characteristics, which were identified to confer aromatase inhibitor (AI) resistance by activating the AKT/mTOR pathway in 2 AI-resistant cell lines (Res-Let cells and Res-Ana cells) (56). The activation of Akt, induced by miR-125b, enhances sensitivity to letrozole and overcomes letrozole resistance in Res-Let cells (56). Downregulated miR-128 results in chemotherapeutic resistance to doxorubicin, through enhancing cell viability and reducing apoptosis and DNA damage in BT-ICs via the modulation of two independent targets, BMI1 and ATP-binding cassette subfamily C member 5 (57). miR-16 has been revealed to be downregulated in BCSCs and to suppress BCSC properties. The overexpression of miR-16 sensitizes MCF-7 cells to doxorubicin by inhibiting Wip1 (Table I) (29).

\section{Prospects}

An increasing number of studies have demonstrated that miRNAs participate in regulating BCSC characteristics via targeting associated genes. miRNAs activate or inactivate multiple signaling pathways by targeting associated genes to effect BCSC formation, self-renewal, differentiation, invasion, metastasis, clonogenicity, tumorigenicity and chemotherapy resistance. BCSCs, as essential drivers of BC metastasis, chemotherapy resistance, relapse and poor prognosis, may be effective therapeutic targets in BC. miRNAs act as critical regulators of $\mathrm{BCSC}$ characteristics, which may provide a novel therapeutic strategy for the treatment of BC. In BCSCs, decreased expression of onco-miRNAs (miR-106b-25, miR-146a, miR-21, miR125, miR-526b, miR-22 and miR-888) or increased expression of anti-onco-miRNAs (miR-140, miR-34, miR-7, miR-16, miR-93, miR-99a and the miR-200 family) may inhibit $\mathrm{BC}$ progression by reducing the levels of expression of oncogenes, while enhancing the levels of expression of anti-oncogenes. Therefore, BCSCs may be potential targets for the miR-based therapy of BC.

\section{Conclusion}

The present review focused on the complicated associations between miRNAs and BCSCs in BC progression. miRNAs, as oncogenes or tumor suppressor genes, may serve pivotal roles in $\mathrm{BC}$ progression by regulating BCSCs, which are a subpopulation of cells that exhibit significant potential for self-renewal, invasion, metastasis and chemoresistance in BC. Several regulatory pathways have been identified, and future studies should be performed to investigate the effects of these regulatory pathways. A comprehensive understanding of the association between BCSCs and miRNAs may provide novel and safer therapeutic strategies for BC.

\section{Acknowledgements}

The present study was supported by a grant from the National Natural Science Foundation of China (grant no. 8157101910).

\section{References}

1. Jeong H, Kim J, Lee Y, Seo JH, Hong SR and Kim A: Neuregulin-1 induces cancer stem cell characteristics in breast cancer cell lines. Oncol Rep 32: 1218-1224, 2014.

2. Gupta PB, Chaffer CL and Weinberg RA: Cancer stem cells: Mirage or reality? Nat Med 15: 1010-1012, 2009.

3. Visvader JE and Lindeman GJ: Cancer stem cells: Current status and evolving complexities. Cell Stem Cell 10: 717-728, 2012.

4. Chen W, Fan XM, Mao L, Zhang JY, Li J, Wu JZ and Tang JH: MicroRNA-224: As a potential target for miR-based therapy of cancer. Tumour Biol 36: 6645-6652, 2015.

5. Zhang D, Zhou P, Wang W, Wang X, Li J, Sun X and Zhang L: MicroRNA-616 promotes the migration, invasion and epithelial-mesenchymal transition of HCC by targeting PTEN. Oncol Rep 35: 366-374, 2016.

6. El Helou R, Pinna G, Cabaud O, Wicinski J, Bhajun R, Guyon L, Rioualen C, Finetti P, Gros A, Mari B, et al: miR-600 acts as a bimodal switch that regulates breast cancer stem cell fate through WNT signaling. Cell Rep 18: 2256-2268, 2017.

7. Liu Y, Zhang J, Sun X, Su Q and You C: Down-regulation of miR-29b in carcinoma associated fibroblasts promotes cell growth and metastasis of breast cancer. Oncotarget 8: 39559-39570, 2017. 
8. Calin GA, Ferracin M, Cimmino A, Di Leva G, Shimizu M, Wojcik SE, Iorio MV, Visone R, Sever NI, Fabbri M, et al: A MicroRNA signature associated with prognosis and progression in chronic lymphocytic leukemia. N Engl J Med 353: 1793-1801, 2005.

9. Fang Y, Xiang J, Chen Z, Gu X, Li Z, Tang F and Zhou Z: miRNA expression profile of colon cancer stem cells compared to non-stem cells using the SW1116 cell line. Oncol Rep 28 2115-2124, 2012

10. Ginestier C, Hur MH, Charafe-Jauffret E, Monville F, Dutcher J, Brown M, Jacquemier J, Viens P, Kleer CG, Liu S, et al: ALDH1 is a marker of normal and malignant human mammary stem cells and a predictor of poor clinical outcome. Cell Stem Cell 1: 555-567, 2007.

11. Dontu G, Abdallah WM, Foley JM, Jackson KW, Clarke MF, Kawamura MJ and Wicha MS: In vitro propagation and transcriptional profiling of human mammary stem/progenitor cells. Genes Dev 17: 1253-1270, 2003.

12. Chang CJ, Chao CH, Xia W, Yang JY, Xiong Y, Li CW, Yu WH, Rehman SK, Hsu JL, Lee HH, et al: p53 regulates epithelial-mesenchymal transition and stem cell properties through modulating miRNAs. Nat Cell Biol 13: 317-323, 2011.

13. van den Beucken T, Koch E, Chu K, Rupaimoole R, Prickaerts P, Adriaens M, Voncken JW, Harris AL, Buffa FM, Haider S, et al: Hypoxia promotes stem cell phenotypes and poor prognosis through epigenetic regulation of DICER. Nat Commun 5: 5203 , 2014.

14. Smith AL, Iwanaga R, Drasin DJ, Micalizzi DS, Vartuli RL, Tan AC and Ford HL: The miR-106b-25 cluster targets Smad7, activates TGF- $\beta$ signaling, and induces EMT and tumor initiating cell characteristics downstream of Six1 in human breast cancer. Oncogene 31: 5162-5171, 2012.

15. Li Q, Yao Y, Eades G, Liu Z, Zhang Y and Zhou Q: Downregulation of miR-140 promotes cancer stem cell formation in basal-like early stage breast cancer. Oncogene 33: 2589-2600, 2014.

16. Kang L, Mao J, Tao Y, Song B, Ma W, Lu Y, Zhao L, Li J, Yang B and Li L: MicroRNA-34a suppresses the breast cancer stem cell-like characteristics by downregulating Notch1 pathway. Cancer Sci 106: 700-708, 2015.

17. Chao $\mathrm{CH}$, Chang CC, Wu MJ, Ko HW, Wang D, Hung MC, Yang JY and Chang CJ: MicroRNA-205 signaling regulates mammary stem cell fate and tumorigenesis. J Clin Invest 124 3093-3106, 2014

18. Zhang H, Cai K, Wang J, Wang X, Cheng K, Shi F, Jiang L, Zhang Y and Dou J: MiR-7, inhibited indirectly by lincRNA HOTAIR, directly inhibits SETDB1 and reverses the EMT of breast cancer stem cells by downregulating the STAT3 pathway. Stem Cells 32: 2858-2868, 2014

19. Wang X, Lu H,Li T, Yu L, Liu G, Peng X and Zhao J: Krüppel-like factor 8 promotes tumorigenic mammary stem cell induction by targeting miR-146a. Am J Cancer Res 3: 356-373, 2013.

20. Han M, Liu M, Wang Y, Chen X, Xu J, Sun Y, Zhao L, Qu H, Fan $\mathrm{Y}$ and $\mathrm{Wu} \mathrm{C}$ : Antagonism of miR-21 reverses epithelial-mesenchymal transition and cancer stem cell phenotype through AKT/ERK1/2 inactivation by targeting PTEN. PLoS One 7: e39520, 2012.

21. Nandy SB, Arumugam A, Subramani R, Pedroza D, Hernandez K, Saltzstein E and Lakshmanaswamy R: MicroRNA-125a influences breast cancer stem cells by targeting leukemia inhibitory factor receptor which regulates the Hippo signaling pathway. Oncotarget 6: 17366-17378, 2015.

22. Yu F, Jiao Y, Zhu Y, Wang Y, Zhu J, Cui X, Liu Y, He Y, Park EY, Zhang $\mathrm{H}$, et al: MicroRNA 34c gene down-regulation via DNA methylation promotes self-renewal and epithelial-mesenchymal transition in breast tumor-initiating cells. J Biol Chem 287: 465-473, 2012

23. Finlay-Schultz J, Cittelly DM, Hendricks P, Patel P, Kabos P, Jacobsen BM, Richer JK and Sartorius CA: Progesterone downregulation of miR-141 contributes to expansion of stem-like breast cancer cells through maintenance of progesterone receptor and Stat5a. Oncogene 34: 3676-3687, 2015

24. Polytarchou C, Iliopoulos D and Struhl K: An integrated transcriptional regulatory circuit that reinforces the breast cancer stem cell state. Proc Natl Acad Sci USA 109: 14470-14475, 2012

25. Iliopoulos D, Lindahl-Allen M, Polytarchou C, Hirsch HA, Tsichlis PN and Struhl K: Loss of miR-200 inhibition of Suz12 leads to polycomb-mediated repression required for the formation and maintenance of cancer stem cells. Mol Cell 39: 761-772, 2010 .
26. Wellner U, Schubert J, Burk UC, Schmalhofer O, Zhu F, Sonntag A, Waldvogel B, Vannier C, Darling D, zur Hausen A, et al: The EMT-activator ZEB1 promotes tumorigenicity by repressing stemness-inhibiting microRNAs. Nat Cell Biol 11: 1487-1495, 2009.

27. Knezevic J, Pfefferle AD, Petrovic I, Greene SB, Perou CM and Rosen JM: Expression of miR-200c in claudin-low breast cancer alters stem cell functionality, enhances chemosensitivity and reduces metastatic potential. Oncogene 34: 5997-6006, 2015.

28. Iliopoulos D, Polytarchou C, Hatziapostolou M, Kottakis F, Maroulakou IG, Struhl K and Tsichlis PN: MicroRNAs differentially regulated by Akt isoforms control EMT and stem cell renewal in cancer cells. Sci Signal 2: ra62, 2009.

29. Zhang X, Wan G, Mlotshwa S, Vance V, Berger FG, Chen H and Lu X: Oncogenic Wipl phosphatase is inhibited by miR-16 in the DNA damage signaling pathway. Cancer Res 70: 7176-7186, 2010.

30. Kastrati I, Canestrari E and Frasor J: PHLDA1 expression is controlled by an estrogen receptor-NFKB-miR-181 regulatory loop and is essential for formation of ER+ mammospheres. Oncogene 34: 2309-2316, 2015.

31. Vazquez-Martin A, Cufí S, López-Bonet E, Corominas-Faja B, Cuyàs $\mathrm{E}$, Vellon L, Iglesias $\mathrm{JM}$, Leis $\mathrm{O}$, Martín $\mathrm{AG}$ and Menendez JA: Reprogramming of non-genomic estrogen signaling by the stemness factor SOX2 enhances the tumor-initiating capacity of breast cancer cells. Cell Cycle 12: 3471-3477, 2013.

32. Zhang Y, Eades G, Yao Y, Li Q and Zhou Q: Estrogen receptor $\alpha$ signaling regulates breast tumor-initiating cells by down-regulating miR-140 which targets the transcription factor SOX2. J Biol Chem 287: 41514-41522, 2012.

33. Liu S, Patel SH, Ginestier C, Ibarra I, Martin-Trevino R, Bai S, McDermott SP, Shang L, Ke J, Ou SJ, et al: MicroRNA93 regulates proliferation and differentiation of normal and malignant breast stem cells. PLoS Genet 8: e1002751, 2012.

34. Liao J, Liu PP, Hou G, Shao J, Yang J, Liu K, Lu W, Wen S, Hu Y and Huang P: Regulation of stem-like cancer cells by glutamine through $\beta$-catenin pathway mediated by redox signaling. Mol Cancer 16: 51, 2017.

35. Yang Z, Han Y, Cheng K, Zhang G and Wang X: miR-99a directly targets the mTOR signalling pathway in breast cancer side population cells. Cell Prolif 47: 587-595, 2014.

36. Majumder M, Landman E, Liu L, Hess D and Lala PK: COX-2 elevates oncogenic miR-526b in breast cancer by EP4 activation. Mol Cancer Res 13: 1022-1033, 2015.

37. Cittelly DM, Finlay-Schultz J, Howe EN, Spoelstra NS, Axlund SD, Hendricks P, Jacobsen BM, Sartorius CA and Richer JK: Progestin suppression of miR-29 potentiates dedifferentiation of breast cancer cells via KLF4. Oncogene 32: 2555-2564, 2013.

38. Shimono Y, Zabala M, Cho RW, Lobo N, Dalerba P, Qian D, Diehn M, Liu H, Panula SP, Chiao E, et al: Downregulation of miRNA-200c links breast cancer stem cells with normal stem cells. Cell 138: 592-603, 2009.

39. Petrelli A, Carollo R, Cargnelutti M, Iovino F, Callari M, Cimino D, Todaro M, Mangiapane LR, Giammona A, Cordova A, et al: By promoting cell differentiation, miR-100 sensitizes basal-like breast cancer stem cells to hormonal therapy. Oncotarget 6: 2315-2330, 2015.

40. Chiotaki R, Polioudaki $\mathrm{H}$ and Theodoropoulos PA: Cancer stem cells in solid and liquid tissues of breast cancer patients: Characterization and therapeutic perspectives. Curr Cancer Drug Targets 15: 256-269, 2015.

41. Han M, Wang Y, Liu M, Bi X, Bao J, Zeng N, Zhu Z, Mo Z, Wu C and Chen X: MiR-21 regulates epithelial-mesenchymal transition phenotype and hypoxia-inducible factor-1 $\alpha$ expression in third-sphere forming breast cancer stem cell-like cells. Cancer Sci 103: 1058-1064, 2012

42. Han M, Liu M, Wang Y, Mo Z, Bi X, Liu Z, Fan Y, Chen X and Wu C: Re-expression of miR-21 contributes to migration and invasion by inducing epithelial-mesenchymal transition consistent with cancer stem cell characteristics in MCF-7 cells. Mol Cell Biochem 363: 427-436, 2012.

43. Song SJ, Poliseno L, Song MS, Ala U, Webster K, Ng C, Beringer G, Brikbak NJ, Yuan X, Cantley LC, et al: MicroRNA-antagonism regulates breast cancer stemness and metastasis via TET-family-dependent chromatin remodeling. Cell 154: 311-324, 2013

44. Lin Y, Liu AY, Fan C, Zheng H, Li Y, Zhang C, Wu S, Yu D, Huang Z, Liu F, et al: MicroRNA-33b inhibits breast cancer metastasis by targeting HMGA2, SALL4 and Twist1. Sci Rep 5: 9995, 2015 
45. Huang S, Cai M, Zheng Y, Zhou L, Wang Q and Chen L: miR-888 in MCF-7 side population sphere cells directly targets E-cadherin. J Genet Genomics 41: 35-42, 2014.

46. Hwang-Verslues WW, Chang PH, Wei PC, Yang CY, Huang CK, Kuo WH, Shew JY, Chang KJ, Lee EY and Lee WH: miR-495 is upregulated by E12/E47 in breast cancer stem cells, and promotes oncogenesis and hypoxia resistance via downregulation of E-cadherin and REDD1. Oncogene 30: 2463-2474, 2011.

47. Ke J, Zhao Z, Hong SH, Bai S, He Z, Malik F, Xu J, Zhou L, Chen W, Martin-Trevino R, et al: Role of microRNA221 in regulating normal mammary epithelial hierarchy and breast cancer stem-like cells. Oncotarget 6: 3709-3721, 2015.

48. Okuda H, Xing F, Pandey PR, Sharma S, Watabe M, Pai SK Mo YY, Iiizumi-Gairani M, Hirota S, Liu Y, et al: miR-7 suppresses brain metastasis of breast cancer stem-like cells by modulating KLF4. Cancer Res 73: 1434-1444, 2013.

49. Jiang F, Li Y, Mu J, Hu C, Zhou M, Wang X, Si L, Ning S and Li Z: Glabridin inhibits cancer stem cell-like properties of human breast cancer cells: An epigenetic regulation of miR-148a/SMAd2 signaling. Mol Carcinog 55: 929-940, 2016.

50. Qian P, Banerjee A, Wu ZS, Zhang X, Wang H, Pandey V, Zhang WJ, Lv XF, Tan S, Lobie PE and Zhu T: Loss of SNAIL regulated miR-128-2 on chromosome $3 \mathrm{p} 22.3$ targets multiple stem cell factors to promote transformation of mammary epithelial cells. Cancer Res 72: 6036-6050, 2012.

51. Chen W, Zhou S, Mao L, Zhang H, Sun D, Zhang J, Li J and Tang JH: Crosstalk between TGF- $\beta$ signaling and miRNAs in breast cancer metastasis. Tumour Biol 37: 10011-10019, 2016.
52. Yin J, Zheng G, Jia X, Zhang Z, Zhang W, Song Y, Xiong Y and He Z: A Bmil-miRNAs cross-talk modulates chemotherapy response to 5-fluorouracil in breast cancer cells. PLoS One 8: e73268, 2013

53. Wu MY, Fu J, Xiao X, Wu J and Wu RC: MiR-34a regulates therapy resistance by targeting HDAC1 and HDAC7 in breast cancer. Cancer Lett 354: 311-319, 2014.

54. Takahashi RU, Miyazaki H, Takeshita F, Yamamoto $\mathrm{Y}$, Minoura K, Ono M, Kodaira M, Tamura K, Mori M and Ochiya T: Loss of microRNA-27b contributes to breast cancer stem cell generation by activating ENPP1. Nat Commun 6: 7318, 2015.

55. Wang HJ, Guo YQ, Tan G, Dong L, Cheng L, Li KJ, Wang ZY and Luo HF: miR-125b regulates side population in breast cancer and confers a chemoresistant phenotype. J Cell Biochem 114: 2248-2257, 2013.

56. Vilquin P, Donini CF, Villedieu M, Grisard E, Corbo L, Bachelot T, Vendrell JA and Cohen PA: MicroRNA-125b upregulation confers aromatase inhibitor resistance and is a novel marker of poor prognosis in breast cancer. Breast Cancer Res 17: 13, 2015.

57. Zhu Y, Yu F, Jiao Y, Feng J, Tang W, Yao H, Gong C, Chen J, Su F, Zhang Y and Song E: Reduced miR-128 in breast tumor-initiating cells induces chemotherapeutic resistance via Bmi-1 and ABCC5. Clin Cancer Res 17: 7105-7115, 2011. 